\title{
Der Zugang zur Ausbildung: Wie integrationsfähig ist das deutsche Berufsbildungssystem?
}

Das berufliche Bildungssystem bereitet in Deutschland eine hohe Zahl junger Menschen auf das Berufsleben vor. Es trägt zu einer geringen Jugendarbeitslosigkeitsrate sowie zum Kompetenzerwerb im jungen Erwachsenenalter bei. Trotz dieser Erfolge - so die zentrale These des Beitrags - hat das deutsche Berufsbildungssystem mittlerweile ein Integrationsproblem, denn etwa jeder siebente junge Erwachsene bleibt ohne Ausbildung. Ursache dafür sind Ausschlussmechanismen im deutschen Berufsbildungssystem. Die Benachteiligung bestimmter Personengruppen kann daher nicht ohne Veränderungen des Systems verringert werden. Deshalb werden auch institutionelle Veränderungen diskutiert, die zu einer erhöhten Integrationskraft beitragen könnten.

HEIKE SOLGA, LAURA MENZE

\section{Integrationskraft von Ausbildungen und Probleme des Ausschlusses}

Die berufliche Ausbildung ist in Deutschland für die meisten jungen Frauen und Männer nach wie vor der wichtigste Weg ins Berufsleben (BMBF 2011). Das deutsche Berufsbildungssystem unterhalb des Hochschulsektors leistet damit einen wichtigen Beitrag zur Integration junger Menschen in den Arbeitsmarkt und die Gesellschaft. Es trägt dazu bei, dass Deutschland im internationalen Vergleich sowohl eine geringe Jugendarbeitslosigkeit als auch einen geringen Anteil an kompetenzarmen Erwachsenen vorweisen kann (OECD 2000, 2010; Solga 2012).

Dieser Erfolg hat jedoch seinen „Preis“, denn „in Ländern mit einem eigenständigen betriebs- und branchenübergreifenden Berufsausbildungssystem [...] werden die Zugangs-, Selektions- und Allokationsprozesse auf dem Arbeitsmarkt wesentlich über die beruflich strukturierten und standardisierten Zertifikate geregelt" (Georg/Sattel 2006, S. 128). Die Forschung weist daher immer wieder darauf hin, dass in Deutschland die zentrale Scheidelinie auf dem Arbeitsmarkt zwischen Personen mit abgeschlossener Berufsausbildung und denen ohne Berufsausbildung verläuft (z. B. Müller/Shavit 1998; Giesecke et al. 2010; OECD 2011, Abbildung A7.1 und A7.2; Solga 2005). Der Zugang zu Ausbildung beeinflusst somit in Deutschland stärker als in anderen Ländern den weiteren Lebens- und
Erwerbsverlauf junger Menschen. All jene, die keine Berufsausbildung abschließen können, sind daher nicht nur kurz-, sondern langfristig benachteiligt.

Bis in die 1980er Jahre hinein gelang es einem immer höheren Anteil an Jugendlichen, eine Ausbildung zu absolvieren (vgl. Baethge et al. 2007; Solga 2005). Seit mehr als zwei Jahrzehnten hat sich allerdings der Anteil der 20- bis 29-Jährigen ohne Berufsausbildung kaum verändert und liegt bei 14 bis $15 \%$ (Tabelle 1). Dies ist individuell wie wirtschaftlich und sozial kein „kleiner“ Anteil, denn er bedeutet, dass etwa jeder siebente junge Mensch ohne Ausbildung bleibt. In absoluten Zahlen sind dies seit Jahren mehr als 1,3 Mio. junge Männer und Frauen.

An der Ausbildungsmotivation der Jugendlichen liegt es häufig nicht. Vielmehr suchen viele Jugendliche nach dem Verlassen der Schule vergeblich einen Ausbildungsplatz. Zum einen gibt es zu wenige Ausbildungsplätze, um alle interessierten Bewerberinnen und Bewerber zu versorgen. Zum anderen wird ein Teil der Jugendlichen als „nicht ausbildungsreif "klassifiziert, sodass Unternehmen sie selbst dann nicht einstellen würden, wenn es mehr Ausbildungsplätze gäbe (siehe Abschnitt 3). Eine sehr hohe Zahl junger Menschen absolviert daher Bildungsgänge des sogenannten Übergangssystems, d.h. berufsvorbereitende Maßnahmen.

Zahlenmäßig sehen die Verhältnisse heute folgendermaßen aus: Knapp die Hälfte der Neuzugänge ins Berufsbildungssystem begann im Jahr 2010 eine betriebliche Ausbildung im dualen System (47,4 \%) (Autorengruppe 
TABELLE 1

Anteil junger Erwachsener ohne abgeschlossene Berufsausbildung - 1996, 2000, 2009

Angaben in Prozent und absoluten Zahlen

\begin{tabular}{cccc}
\hline 20- bis $\begin{array}{c}\text { 24-Jährige } \\
\text { in \% }\end{array}$ & in \% & 20- bis 29-Jährige \\
absolut in Mio.
\end{tabular}

mentation des Ausbildungsmarktes in Berufsausbildungen, die unterschiedliche Eingangsqualifikationen voraussetzen (vgl. Protsch 2011 sowie dies. in diesem Heft), sowie die Unterteilung des Berufsbildungssystems in schulische und duale Ausbildungsgänge, die zumeist für unterschiedliche Berufe ausbilden (also nicht alternative Ausbildungswege zum gleichen Beruf darstellen) und zudem unterschiedliche Zugänge zu Ausbildungen und Berufen für junge Männer und Frauen strukturieren. Und schließlich sind selbst die Bildungsangebote im Übergangssystem segregiert und tragen zu einer Verstetigung ungleicher Ausbildungschancen bei (siehe Abschnitt 4). Diese Ausgrenzungsmechanismen sind der derzeitigen Organisation des deutschen Berufsbildungssystems immanent, sodass das Insider-Outsider-Problem und damit die Benachteiligung bestimmter Personengruppen (siehe Abschnitt 3) - zum Teil verschärft durch berufsstrukturelle Entwicklungen (Abschnitt 5) - ohne Veränderungen des Systems nicht beseitigt werden können. Welche institutionellen Veränderungen dafür erforderlich wären, wird in Abschnitt 6 beispielhaft diskutiert.

Vor dem Hintergrund dieses Insider-Outsider- oder Integrationsproblems lauten die beiden zentralen Fragen des Beitrags:

(1) Inwieweit gelingt möglichst vielen der Zugang zur Ausbildung (oder umgekehrt: Wie viele müssen ohne Ausbildung bleiben)?

(2) Wie werden die Zugangschancen strukturiert, d.h. welche sozialen Ungleichheiten bestehen hier hinsichtlich Schulbiografie, sozialer Herkunft, Geschlecht oder Migrationshintergrund?

In Bezug auf die erste Frage wurde oben bereits gezeigt, dass es einerseits trotz schwankender Nachfragen und zum Teil nachteiliger wirtschaftlicher Bedingungen gelungen ist, ein „stabiles Ergebnis“ zu produzieren. Die Zahl der Jugendlichen

\section{Thesen und Fragestellungen}

In der Bilanz heißt das zunächst: Das berufliche Bildungssystem spielt weiterhin eine sehr wichtige Rolle für die Vorbereitung auf das Berufsleben. Bei allen Erfolgen - so die zentrale These des Beitrags - besteht im deutschen Berufsbildungssystem jedoch zugleich ein Insider-Outsider-Problem für diejenigen, die die „Eintrittskarte“ zum beruflich strukturierten Arbeitsmarkt nicht erworben haben (vgl. auch Müller/Shavit 1998). So ergibt sich eine Knappheit des Angebots an Ausbildungsplätzen dadurch, dass Ausbildungsplätze durch einen Ausbildungsmarkt bereitgestellt werden und der Nachfrageüberhang seitens der Jugendlichen durch das Übergangssystem aufgefangen wird - statt alternative, beruflich voll qualifizierende Ausbildungsangebote (z. B. an Berufsschulen) anzubieten. Diese Knappheit sichert eine Monopolstellung derjenigen, die es auf einen Ausbildungsplatz geschafft haben (der Insider), bzw. bedeutet einen langfristigen Ausschluss jener, denen dies nicht gelungen ist (der Outsider). Verschärft wird dieses Problem durch die Seg-
Seit 2009 liefert der Nationale Bildungsbericht auch korrigierte Werte, die Doppelzählungen zwischen Schulstatistik und Maßnahmen der Bundesagentur für Arbeit identifizieren. Bei den oben genannten Angaben handelt es sich um die unkorrigierten Werte, damit Vergleiche zu den Vorjahren möglich sind. Die Anteile ohne Doppelzählungen weichen allerdings nur marginal von den unkorrigierten Werten ab (für 2010: 48,9 \% im dualen System, 20,4 \% im Schulberufssystem und $30,7 \%$ im Übergangssystem).

(2) Die Abnahme der absoluten Zahlen ist kleineren Geburtsjahrgängen geschuldet. Ferner gibt es große Unterschiede zwischen den Bundesländern. In einigen westdeutschen Flächenländern, wie Baden-Württemberg, Niedersachsen oder bei circa $40 \%$ (Autorengruppe Bildungsberichterstattung 2012, Abbildung E1-7web). In den neuen Bundesländern betrug er hingegen zumeist weniger als $20 \%$, da hier vermehrt öffentlich finanzierte außerbetriebliche Ausbildungsplätze bereitgestellt wurden (Baethge 2008; Pötter et al. 2012). Schleswig-Holstein, lag der Anteil des Übergangssystems 
ohne Ausbildungsabschluss hätte sich angesichts geburtenstarker Jahrgänge, der Wiedervereinigung sowie einer wirtschaftlich angespannten Lage in den letzten zwei Jahrzehnten auch deutlich vergrößern können. Dies ist gleichwohl nicht geschehen - und daher als Stärke des deutschen Berufsbildungssystem zu werten. Andererseits wurde deutlich, dass das deutsche Ausbildungssystem (derzeit) weit weniger jungen Menschen eine voll qualifizierende Berufsausbildung geben kann, als diese nachgefragt wird. Das Übergangssystem verharrt auch bei abnehmenden Jahrgangsstärken auf einem sehr hohen Niveau (wie die Zahlen für 2010 zeigen). Der Markt allein kann daher eine vollständige Integration aller ausbildungssuchenden Jugendlichen nicht gewährleisten, und das Schulberufssystem ist (bisher) nicht derart ausgestaltet, dass es erfolglosen Jugendlichen - sei es aufgrund von (regionalen) Marktungleichgewichten oder aufgrund von Leistungsmerkmalen, die ihre Auswahl seitens der Betriebe be- oder verhindern (siehe Abschnitt 3) - eine voll qualifizierende Alternative anbietet. Im Folgenden werden wir daher die zweite Frage nach der sozialen Strukturierung von Ungleichheiten beim Ausbildungszugang behandeln.

\section{Chancenungleichheiten im Ausbildungssystem}

In der soziologischen Bildungsforschung werden ungleiche Chancen beim Zugang zur Ausbildung üblicherweise hinsichtlich der sozialen Herkunft, der Geschlechterzugehörigkeit und des Migrationshintergrunds betrachtet. Ungleiche Chancen entlang dieser Faktoren werden als nicht meritokratisch oder nicht gerecht definiert, da sie nicht auf der eigenen Leistung, sondern den sozialen Zugehörigkeiten qua Geburt beruhen.

Wir werden darüber hinaus auch Ungleichheiten entlang der schulischen Vorbildung als Verstoß gegen Chancengleichheit betrachten. Da die deutsche Schule die Aufgabe hat, Jugendliche für das Arbeitsleben vorzubereiten und ihnen die notwendigen Einstiegsqualifikationen für eine Ausbildung zu vermitteln, werden wir danach fragen, inwieweit den Schulabgängerinnen und -abgängern aus den unterschiedlichen Bildungsgängen der Übergang in eine Ausbildung gelingt. Gelingt es nicht, ist zu fragen, inwiefern Vorbehalte der auswählenden Akteure im Ausbildungssystem hinsichtlich der kognitiven Leistungspotenziale von Ausbildungssuchenden mit unterschiedlichen Schulabschlüssen - entgegen der oben genannten Aufgabe von Schule - eine Ursache dieses Misserfolgs sind.

\subsection{Ungleichheiten nach schulischer Vorbildung}

Bei Chancengleichheit geht es nicht darum, ob alle Schulabgängerinnen und -abgänger zu den gleichen Ausbildungs-
ABB. 1

\section{Verteilung der Neuzugänge auf die drei Sektoren des beruflichen Ausbildungssystems nach schulischer Vorbildung 2010}

Angaben in Prozent

Übergangssystem

Schulberufssystem

Duales System

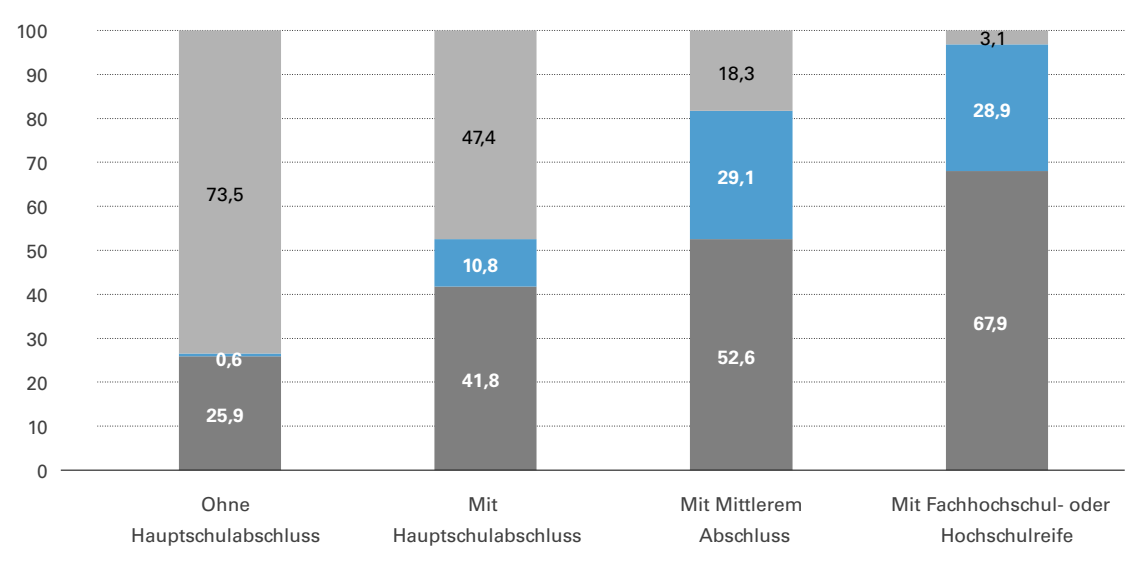

Quelle: Autorengruppe Bildungsberichterstattung (2012), Abb. E1-8web, ohne Doppelzählungen.

berufen Zugang haben, sondern darum, ob sie überhaupt in eine voll qualifizierende Ausbildung gelangen können. Ist Letzteres nicht der Fall, so ergeben sich Zweifel an der Fähigkeit des deutschen Schulsystems, die notwendigen Grundlagen für eine Ausbildung zu vermitteln und gleiche Startbedingungen für die Suche nach einem Ausbildungsplatz zu schaffen. Zudem wäre dann auch die quantitative und qualitative Ausgestaltung des deutschen Berufsbildungssystems zu hinterfragen. Das heißt, es gäbe generell zu wenige Ausbildungsplätze und insbesondere zu wenige voll qualifizierende Ausbildungsangebote (in Betrieben, Berufsschulen oder bei freien Trägern), die es auch Jugendlichen mit schlechteren Schulleistungen ermöglichen, sich adäquat auf einen Beruf vorzubereiten, um auf dem deutschen beruflich strukturierten Arbeitsmarkt bestehen zu können.

Wie Abbildung 1 zeigt, unterscheiden sich die Ausbildungschancen deutlich nach dem schulischen Vorbildungsniveau der Jugendlichen. Besonders schlecht sind die Aussichten von Ausbildungssuchenden ohne Schulabschluss. Hiervon schafften 2010 lediglich 26,5\% den Übergang in eine Ausbildung (überwiegend im dualen System). Fast acht von zehn dieser Jugendlichen (73,5\%) landeten jedoch im Übergangssystem. Auch bei den Ausbildungssuchenden mit einem Hauptschulabschluss fand nur etwas mehr als die Hälfte einen Ausbildungsplatz. Für knapp die Hälfte (47,4 \%) von ihnen gab es allerdings auch nur ein Angebot im Übergangssystem.

Für jene mit einem mittlerem Schulabschluss sah die Situation deutlich besser aus. Hier fand der größte Teil eine voll qualifizierende Ausbildung (81,7\%). Doch selbst bei ihnen landete fast jeder Fünfte im Übergangssystem 
$(18,3 \%){ }^{3}$ Ausbildungssuchende mit Studienberechtigung hatten sehr gute Zugangschancen zu voll qualifizierenden Ausbildungsplätzen. Nur 3,1 \% von ihnen mündeten in das Übergangssystem ein. ${ }^{\circ}$

Die hohe Quote der Ausbildungssuchenden mit einem mittleren Schulabschluss im Übergangssystem zeigt die quantitativen Engpässe bei der (regionalen) Bereitstellung von Ausbildungsplätzen in Deutschland, da zumindest hier von einer vorhandenen „Ausbildungsreife“ auszugehen ist. Diese jungen Menschen werden daher häufig auch als "Marktbenachteiligte“ bezeichnet.

Bei nicht erfolgreichen Ausbildungssuchenden mit maximal einem Hauptschulabschluss wird jedoch zumeist eine mangelnde oder gar (noch) fehlende "Ausbildungsreife“ unterstellt. Weit weniger wird hier das Fehlen von Ausbildungsplätzen als das Kernproblem des Übergangs in eine voll qualifizierende Ausbildung gesehen. So hieß es zum Ausbildungspakt 2009: „[...] die Paktpartner stehen auch in diesen schwierigeren Zeiten zu ihrer Paktzusage, jedem ausbildungswilligen und -fähigen Jugendlichen ein Ausbildungsangebot zu machen. Die aktuellen Daten der BA zeigen, dass dies gelingt.“ (Pressemitteilung zum Ausbildungspakt 2009, 13.10.2009) Damit werden jene, denen es nicht gelungen ist, eine Ausbildungsstelle zu finden, als (noch) „nicht ausbildungsreif“ eingestuft. Im Ausbildungspakt 2010 wurde dementsprechend dann formuliert: „[...] der immer noch hohe Anteil an Jugendlichen mit Schwierigkeiten beim Schulabschluss und bei der Einmündung in Ausbildung erforder[ $\mathrm{t}$ ] deutliche Verbesserungen in der Ausbildungsreife und Berufsorientierung [...]" (Nationaler Pakt für Ausbildung und Fachkräftenachwuchs in Deutschland 2010). Und auch im Ausbildungspakt 2012 lautet eine der drei zentralen Paktzusagen „Ausbildungsreife sicherstellen und Berufsorientierung verbessern“ (Nationaler Pakt für Ausbildung und Fachkräftenachwuchs in Deutschland 2012).

Die „Ausbildungsreife“ von Jugendlichen kann allerdings nicht von vornherein qua Schulabschluss bestimmt werden. So gelingt einem substanziellen Anteil an Jugendlichen mit maximal einem Hauptschabschluss der Übergang in eine Ausbildung (Abbildung 1). Ferner zeigen Untersuchungen, dass darunter durchaus auch Jugendliche mit mangelnden kognitiven und nicht-kognitiven Fähigkeiten sind (Kohlrausch/Solga 2012; Solga et al. 2012). Die Bestimmung von "Ausbildungsreife“ ist somit kontextabhängig von der jeweiligen Ausbildung und den einstellenden Ausbildungsbetrieben.

Zudem ist spätestens mit den Veröffentlichungen der Ergebnisse der ersten Studie des Programme for International Student Assessment (PISA) im Jahr 2001 auch im öffentlichen Bewusstsein bekannt, dass die Chancen im allgemeinbildenden Schulsystem und damit für den individuellen Schulerfolg stark nach der sozialen Herkunft und dem Migrationshintergrund strukturiert sind - und nur zum Teil durch Unterschiede in den individuellen Kompetenzen (siehe die zahlreichen Veröffentlichungen zu den PISA-Studien) oder in den kognitiven Lernpotenzialen (vgl. auch Uhlig et al. 2009; Uhlig 2010). Ungleiche Chancen im allgemeinbildenden Schulsystem werden somit beim Übergang ins Berufsbildungssystem fortgeschrieben (siehe auch Protsch/Dieckhoff 2011, 2012). Das deutsche Berufsbildungssystem trägt insofern (durch seinen ausgeprägten Marktmechanismus) dazu bei, dass früh im Lebensverlauf erzeugte Bildungsungleichheiten im Ausbildungssystem und damit verbunden im späteren Erwerbsleben (insbesondere mit Blick auf das Arbeitslosigkeitsrisiko) weiter fortbestehen und sich verfestigen.

\subsection{Ungleichheiten nach Migrations- hintergrund}

Jugendliche mit Migrationshintergrund haben deutlich geringere Chancen, einen Ausbildungsplatz zu finden, als Ausbildungssuchende ohne Migrationshintergrund. Diese Benachteiligung lässt sich nur zum Teil durch ihr im Durchschnitt niedrigeres Vorbildungsniveau und ihre ungünstigeren sozialen Herkunftsressourcen erklären. So belegen empirische Untersuchungen ${ }^{\ominus}$ einheitlich, dass auch unter Kontrolle dieser Faktoren Ausbildungssuchende mit Migrationshintergrund deutlich schlechtere Ausbildungschancen haben: „[...] junge Frauen wie Männer mit Migrationshintergrund [haben] selbst unter den gleichen Voraussetzungen in Bezug auf Schulabschluss, Schulnoten, soziale Herkunft und soziale Einbindung schlechtere Chancen [...], einen betrieblichen bzw. vollqualifizierenden Ausbildungsplatz zu erhalten, als junge Frauen und Männer ohne Migrationshintergrund.“ (BIBB 2011, S. 192; vgl. auch BIBB 2012, S. 188)

Dabei „lässt sich zeigen, dass die ungünstige Position von Jugendlichen mit Migrationsgeschichte beim Eintritt in eine berufliche Ausbildung ausschließlich den Einstellungspraktiken auf dem dualen Ausbildungsstellenmarkt zu verdanken ist. Denn werden nur die Eingangsqualifikationen von Jugendlichen, die eine Berufsfachschule besuchen, betrachtet, so gibt es hier bei gleicher allgemeiner Fachleistung zwischen Deutschen mit deutscher Muttersprache und ausländischen Jugendlichen überhaupt keinen Vorteil für eine der beiden Gruppen, und die niedrigere kritische Schwelle für Deutsche mit nichtdeutscher Herkunftssprache ist gering." (Lehmann et al. 2006, S. 183) Dies verdeutlicht, dass ihre Ausbildungschancen vor allem durch

(3) Schulabgänger mit mittlerem Abschluss, die auf einer beruflichen Schule die Studienberechtigung erwerben, zählen dabei nicht als Teilnehmende im Übergangssystem.

(4) Nicht zu vergessen ist dabei, dass erfolglosen Studienberechtigten - im Unterschied zu den anderen erfolglosen Jugendlichen - auch der Weg an eine Hochschule offen steht.

(5) Siehe z. B.: BA/BIBB-Bewerberbefragung (BIBB 2011); BIBBÜbergangspanel (Beicht/Granato 2010); Lehmann et al. 2005 und Lehmann et al. 2006. 
den Marktmechanismus der dualen Ausbildung geringer sind, d. h. dass ihr Migrationshintergrund hier - über den Schulabschluss hinaus - eine besondere Relevanz bei Auswahlentscheidungen erhält. Studien belegen, dass eine wichtige Ursache dafür ein diskriminierendes Rekrutierungsverhalten von (Ausbildungs-)Betrieben ist (z. B. Bertrand/ Mullainathan 2004; Goldberg et al. 1995).

Hinsichtlich der vorzufindenden Chancenungleichheiten sind dabei zwei Merkmale des Migrationshintergrundes von besonderer Bedeutung. Zum einen haben Ausbildungssuchende, die nicht die deutsche Staatsangehörigkeit besitzen, nochmals schlechtere Ausbildungschancen als Migrantinnen und Migranten mit deutschem Pass. Zum anderen belegen die Ergebnisse von Untersuchungen zu Leistungen, Motivation und Einstellungen beim Ausbildungsbeginn (ULME I, vgl. Lehmann et al. 2005) unterschiedliche Ausbildungschancen in Abhängigkeit vom Herkunftsland. Bei gleichen individuellen Fachleistungen haben Jugendliche aus der Türkei, dem Iran sowie aus Afghanistan besonders schlechte Aussichten, einen Ausbildungsplatz zu erhalten (ebd., S. 112).

\subsection{Ungleichheiten nach Geschlecht und sozialer Herkunft}

Des Weiteren strukturieren die Faktoren Geschlecht und soziale Herkunft den Zugang zu voll qualifizierenden Ausbildungsgängen. Junge Männer sind häufiger im Übergangssystem zu finden als junge Frauen (2010: 58 versus $42 \%$; Autorengruppe Bildungsberichterstattung 2012, Tabelle E1 - 3web). Eine Ursache dafür ist der im Durchschnitt geringere Schulerfolg von Jungen im Vergleich zu Mädchen sowie der größere Anteil an Berufswünschen, die nur im dualen System und damit „marktabhängig“ angeboten werden. Mädchen haben häufiger Berufswünsche, die mit einer Ausbildung im Schulberufssystem verwirklicht werden können - die allerdings zumeist den mittleren Schulabschluss und zum Teil sogar ein Abitur voraussetzen.

Jugendliche aus sozial benachteiligten Familien sowie aus Elternhäusern, in denen kein Elternteil eine Ausbildung (oder ein Studium) absolviert hat, haben ebenfalls geringere Ausbildungschancen (Beicht/Granato 2010; Lehmann et al. 2005; Solga et al. 2012). Für sie besteht das Problem weniger darin, dass sie nicht wissen, was sie werden wollen oder was die Anforderungen an bestimmte Berufe sind. Sie haben durchaus konkrete und realistische Berufsvorstellungen (vgl. Kohlrausch/Solga 2012; Solga et al. 2012). Barrieren gibt es vielmehr bei der Bewerbung, da ihre Eltern sie weniger gut bei der schriftlichen Bewerbung und in der Vorbereitung auf Vorstellungsgespräche unterstützen können. Zudem sind ihre Eltern häufiger arbeitslos, sodass sie weniger von Rekrutierungsnetzwerken (z. B. als „Mitarbeiterkind“) profitieren können (vgl. Dietz et al. 2011; Koch 1993, S. 9; Seyfried 2006, S. 35).

Zusammenfassend zeigt sich, dass beim Übergang in die Ausbildung vielfältige soziale Ungleichheiten (fort-) bestehen, die bereits im allgemeinbildenden Schulsystem zu ungleichen Bildungschancen geführt haben. Insofern gelingt es zwar dem deutschen Berufsbildungssystem, dass viele junge Menschen mit einer beruflichen Ausbildung den Weg ins Erwachsenenleben beschreiten, gleichwohl ist dessen Integrationskraft gerade für sozial benachteiligte und leistungsschwächere Gruppen stark eingeschränkt.

\section{Integration über das Übergangs- system?}

Baethge (2008, S. 568) resümiert zum Übergangssystem: „Mit dem Ausbau des Übergangssystems wurde zwar eine hohe Integration geringqualifizierter Jugendlicher in das berufliche Bildungssystem erreicht - allerdings einhergehend mit einer starken institutionellen Ausgliederung dieser sozial und marktbenachteiligten Jugendlichen in ein Parallelsystem.“ Angesichts solcher „institutionellen Ausgliederung“ ist zu fragen, ob diese Integration „nur“ darin besteht, Jugendliche und junge Erwachsene vor Arbeitslosigkeit zu schützen, oder ob junge Menschen nach dem Absolvieren von Maßnahmen tatsächlich bessere Möglichkeiten haben, in eine voll qualifizierende Ausbildung zu wechseln. Zumindest in systemischer Hinsicht ist Letzteres nicht gegeben, weil die Maßnahmen des Übergangssystems vollkommen unabhängig vom Ausbildungssystem laufen (z.B. nicht mit der Zusage einer späteren Ausbildung verbunden sind). Der erfolgreiche Abschluss einer Maßnahme stellt somit weder eine notwendige noch eine hinreichende Bedingung für die Verbesserung der Ausbildungschancen dar.

Ob diese Maßnahmen daher (wenigstens) zu einer Verbesserung der individuellen Voraussetzungen der Jugendlichen beitragen und damit ihre Chancen auf dem Ausbildungsmarkt in der Konkurrenz mit anderen tatsächlich erhöhen können, wird in der Literatur unter dem Stichwort „Maßnahmenkarrieren“ gleichfalls kritisch diskutiert (z. B. Baethge et al. 2007, S. 51; Krekel/Ulrich 2009). So zeigen beispielsweise Ergebnisse des Hauptschulpanels des Deutschen Jugendinstituts (DJI), dass ein Drittel der Hauptschülerinnen und Hauptschüler, die nach der Schulzeit in das Übergangssystem eintraten, im Anschluss eine weitere berufsvorbereitende Maßnahme absolviert haben (Reißig et al. 2008a). Auch zwei Jahre nach Verlassen der Schule war nur die Hälfte von ihnen in einer voll qualifizierenden Ausbildung. Besonders bedenklich ist, dass sich rund $15 \%$ dieser Jugendlichen (also etwa jeder Sechste) zu diesem Zeitpunkt weder in Ausbildung noch in Beschäftigung befanden (ReiBig et al. 2008b).

Zudem ist das Übergangssystem nicht „inklusiv“, sondern selbst wiederum (leistungsmäßig und sozial) segregierend organisiert, sodass bestehende Unterschiede aus der Schulzeit beim Zugang zu den unterschiedlichen Bil- 
dungsangeboten des Übergangssystems fortgeschrieben werden. Jugendliche werden hier nach dem Niveau ihres Schulabschlusses in unterschiedliche Bildungsgänge sortiert. So nehmen am Berufsvorbereitungsjahr (BVJ) und berufsvorbereitenden Maßnahmen der Bundesagentur für Arbeit (BvB) vor allem Jugendliche ohne Schulabschluss und Abgänger aus Förderschulen teil, die zudem oft aus bildungsschwachen Familien stammen. Die Teilnehmenden des Berufsgrundbildungsjahres (BGJ) besitzen hingegen zumeist einen Hauptschulabschluss und können in der Regel bessere Schulnoten vorweisen als die Jugendlichen im BVJ. Die ein- oder zweijährigen teilqualifizierenden Bildungsgänge an Berufsfachschulen (BFS) werden wiederum von Jugendlichen mit nochmals besseren Ausgangsbedingungen besucht. Zum Großteil verfügen sie über einen mittleren Schulabschluss und gute Schulnoten. ${ }^{\ominus}$ Im Gegensatz zu den überwiegend von jungen Männern besuchten Maßnahmen des BVJ, des BGJ und der BvB sind in den teilqualifizierenden Angeboten an Berufsfachschulen zu über $50 \%$ junge Frauen zu finden (Beicht 2009).

Beicht (2009) zeigt auf Basis des Übergangspanels des Bundesinstituts für Berufsbildung, dass diese Segregation mit unterschiedlichen Möglichkeiten der Chancenverbesserung für die Teilnehmenden einhergeht: Im BVJ/BvB können $12 \%$ der gering vorgebildeten Jugendlichen einen Schulabschluss (in der Regel den Hauptschulabschluss) erwerben. Im BGJ holen die bereits höher Vorgebildeten zu jeweils etwa $5 \%$ einen Hauptschul- bzw. einen mittleren Abschluss nach. In den teilqualifizierenden Bildungsgängen der Berufsfachschulen erreicht wiederum gut die Hälfte (51\%) der bereits besser vorgebildeten Jugendlichen einen höheren Schulabschluss. In der Regel handelt es sich um einen mittleren Abschluss (34\%) oder das (Fach-)Abitur $(16 \%){ }^{\ominus}$ Im Ergebnis sind „die Unterschiede in der Verteilung der Schulabschlüsse [...] nach der Beendigung der Übergangsmaßnahmen noch größer als vorher" (Beicht 2009, S. 9). Soziale Ungleichheiten des allgemeinbildenden Schulsystems werden somit im Übergangssystem eher verstärkt als abgebaut und Ungleichheiten in den Ausbildungsmarktchancen erneut bekräftigt.

\section{Herausforderungen für die Integrationsfähigkeit des deutschen Berufsbildungssystems}

Diese Ungleichheiten beim Zugang zur Ausbildung sind vor dem Hintergrund gegenläufiger Entwicklungen zu sehen, die das Berufsbildungssystem und seine Integrationsfähigkeit in der Vergangenheit bereits herausgefordert haben und in der Zukunft noch stärker herausfordern werden: Zum einen gibt es angesichts der demografischen Entwicklung eine stark abnehmende Nachfrage nach beruflicher
Ausbildung - bei einem wohl gleichbleibenden Anteil an Jugendlichen mit geringer schulischer Vorbildung. Zum anderen steigen zum Teil die Qualifikationsanforderungen der Arbeitswelt und damit einhergehend der jeweiligen Ausbildungsberufe.

\subsection{Demografische Entwicklungen}

In Ostdeutschland verlassen bereits heute sehr geburtenschwache Jahrgänge das Schulsystem; in Westdeutschland ist ab 2014 von einem Rückgang der Abgängerzahlen auszugehen (vgl. Krekel/Ulrich 2009). Für die kommenden Jahre zeichnet sich neben diesen quantitativen Veränderungen in der Nachfrage nach beruflicher Ausbildung seitens der Jugendlichen auch eine Reihe qualitativer Veränderungen $\mathrm{ab}$.

Zum einen wird die Zahl der Ausbildungssuchenden zwischen 2010 und 2020 um insgesamt 50.000 zurückgehen, wobei der Anteil mit maximal einem Hauptschulabschluss unter ihnen - wie heute - weiterhin bei circa $35 \%$ liegen dürfte (BIBB 2011, Tabelle A2.2.2 -1). Der zahlenmäßig stärkste Rückgang ist bei Jugendlichen mit mittlerem Schulabschluss zu erwarten; hier wird die Nachfrage in diesem Zeitraum um etwa 27.000 Interessenten (von 196.689 auf 169.528 bzw. um $14 \%$ ) sinken. Ferner wird der Anteil an Studienberechtigten, die potenziell ein Studium statt einer Ausbildung anstreben, steigen. ${ }^{8}$ Die Wirtschaft muss sich daher auch zukünftig auf einen hohen Anteil eher gering qualifizierter Ausbildungssuchender einstellen - denen anders als heute kein ausreichendes Potenzial an höher qualifizierten Ausbildungssuchenden gegenüberstehen wird, um die Nachfrage seitens der Betriebe zu befriedigen. In bestimmten Regionen - insbesondere in Ostdeutschland ist dies bereits heute der Fall. Zukünftig wird dieses Problem auch zahlreiche weitere Regionen in Westdeutschland betreffen.

Zum anderen wird der Anteil an Jugendlichen mit Migrationshintergrund stark ansteigen. So liegt dieser Anteil unter den 15- bis 20-Jährigen derzeit bei $26 \%$, in der Altersgruppe der heute 10- bis 15-Jährigen sind es bereits $29 \%$ und unter den 0- bis 5-Jährigen sogar $35 \%$ (Statistisches

(6) Zur schulischen Vorbildung derTeilnehmenden verschiedener Maßnahmen des Übergangssystems siehe Statistisches Bundesamt (2011b) sowie für Baden-Württemberg das Gutachten des Expertenrats „Herkunft und Bildungserfolg" (2011).

(7) Bildungsgänge an Berufsschulen, die zu einem Abitur führen, sollten allerdings nicht als Teilnahme am Übergangssystem gewertet werden, sondern positiv - wie in der Bildungsberichterstattung üblich - als Erhöhung der Durchlässigkeit des Berufsbildungssystems.

(8) Nicht berücksichtigt sind in den Projektionen des BIBB die möglichen Auswirkungen der gegenwärtigen Sekundarschulreformen in zahlreichen Bundesländern. 
Bundesamt 2011a). In zahlreichen Bundesländern und Regionen wird dies noch stärker der Fall sein, so z. B. in BadenWürttemberg, wo der Migrantenanteil unter den 0- bis unter 5-Jährigen im Jahr 2010 bei $41 \%$ lag (Expertenrat „Herkunft und Bildungserfolg“ 2011, S. 8).

Oftmals wird in der Abnahme der Jahrgangsstärken die „Lösung" des derzeitigen Ausbildungsplatzmangels und der geringen Ausbildungschancen von Jugendlichen mit geringen Schulleistungen (häufig Jungen) oder mit Migrationshintergrund gesehen. Mit einer insgesamt verringerten Bewerberzahl gegenüber den vorhandenen Ausbildungsplätzen wird teilweise davon ausgegangen, dass sich das Übergangssystem quasi „von selbst“ abbauen wird (z.B. Krekel/Ulrich 2009, S. 26). Für die (zahlenmäßig stark abnehmende) Gruppe der Ausbildungssuchenden mit einem mittleren Schulabschluss (die als „marktbenachteiligt“ gelten) könnte dies eine zutreffende Prognose sein. Fraglich ist jedoch, ob dies auch für Ausbildungssuchende gilt, bei denen von einer zu geringen oder gar fehlenden „Ausbildungsreife" ausgegangen wird. Gleichfalls ist offen, ob die Diskriminierung junger Migrantinnen und Migranten bei der Suche nach betrieblichen Ausbildungsplätzen tatsächlich abnehmen wird.

Damit auch diese beiden Gruppen von der oben dargestellten quantitativ zurückgehenden Ausbildungsnachfrage „profitieren“ können, müssten Betriebe ihre Rekrutierungspraxen und -kriterien im Vergleich zu heute ändern, sodass die einen nicht qua Schulabschluss und die anderen nicht qua Migrationshintergrund aussortiert werden. Ob dies geschehen wird, ist fraglich - automatisch wird es wohl nicht passieren. So lassen Betriebe derzeit eine Ausbildungsstelle eher unbesetzt, als sie weniger qualifizierten Bewerbern und Bewerberinnen zu geben (Gericke et al. 2009). Zudem haben Migrantinnen und Migranten auch heute bei guten Schulabschlüssen und -leistungen schlechte(re) Ausbildungschancen (siehe Abschnitt 3).

\subsection{Wirtschaftlicher und technologischer Wandel}

Vorhandene Ausbildungsplätze eher unbesetzt zu lassen, wird seitens der Betriebe (und ihrer Verbände) zumeist mit dem Verweis auf höhere Anforderungen in der Arbeitswelt begründet. Es ist unbestreitbar, dass sich sowohl sektoral als auch tätigkeitsbezogen die Qualifikationsanforderungen der Arbeitswelt verändert haben und weiter verändern werden. Wie das Gutachten der Expertenkommission Forschung und Innovation (EFI) 2009 ausweist, ist der Anteil der Beschäftigten im nicht-wissensintensiven produzierenden Gewerbe zwischen 1991 und 2007 um ca. ein Drittel zurückgegangen, während der Anteil der Beschäftigten in den wissensintensiven Dienstleistungen um ca. $30 \%$ gestiegen ist (EFI 2009, S. 57). Diese Entwicklung wird sich, wenn auch in geringerem Umfang als in der Vergangenheit, weiter fortsetzen (vgl. Autorengruppe Bildungsberichterstattung 2010, S. 163; Schnur/Zika 2007).
Im Ergebnis zeigt sich einerseits ein Schrumpfen der Beschäftigungszahlen im industriellen Bereich, in dem auch Jugendliche mit niedrigen Schulabschlüssen noch Zugangsmöglichkeiten zu (dualen) Ausbildungen haben. Andererseits nehmen die Beschäftigtenzahlen im wissensintensiven Dienstleistungsbereich zu - und damit in jenem Bereich, der bei der Besetzung von Ausbildungsplätzen an sehr guten mittleren Schulabschlüssen oder gar dem Abitur orientiert ist.

Die dargestellten sektoralen und berufsstrukturellen Entwicklungen werfen zwei Fragen auf:

(1) Inwieweit sind mit diesen Entwicklungen tatsächlich höhere Anforderungen an die schulische Vorbildung der Auszubildenden verbunden?

(2) Inwieweit fehlen den schulisch gering qualifizierten Jugendlichen die erforderlichen Kompetenzen, um mit diesen Höherentwicklungen der Arbeitswelt Schritt zu halten?

Hinsichtlich der qualifikatorischen Anforderungen der Ausbildungsberufe gibt es unterschiedliche Entwicklungen. So konnte Protsch (2011) auf der Grundlage von Inhaltsanalysen von Ausbildungsrahmenplänen zeigen, dass sich in manchen Berufsbereichen tatsächlich die Komplexität der Arbeits- und Ausbildungsanforderungen erhöht hat, so z. B. bei den Medizinischen Fachangestellten. Diese sogenannten Upskilling-Prozesse haben jedoch nicht in allen Berufen stattgefunden. In manchen Bereichen hat sich die Komplexität der Ausbildungsinhalte nicht verändert, beispielsweise für den Beruf des Bürokaufmanns bzw. der Bürokauffrau. Hier haben eher informale Upgrading-Prozesse stattgefunden, d.h. viele Arbeitgeber haben sich aufgrund des hohen Angebots an Auszubildenden an einen mittleren Abschluss "gewöhnt" und setzen diesen Abschluss heute voraus. Jugendliche mit einem Hauptschulabschluss werden in entsprechenden Rekrutierungsprozessen häufig nicht mehr in Betracht gezogen, obgleich sie diese Berufe durchaus erlernen und ausüben könnten.

Gleichwohl ist hinsichtlich der aus der Schule mitgebrachten Kompetenzen bei einer substanziellen Zahl von Hauptschülerinnen bzw. Hauptschülern durchaus von Defiziten auszugehen, wie die Ergebnisse der PISA-Studien immer wieder zeigen. Eine der Ursachen dafür ist, dass die Lehrpläne für Hauptschulen wenig(er) anspruchsvoll sind. Ein weiterer Grund ist, dass der Lernkontext in Hauptschulen teilweise derart ungünstig ist, dass ein lernförderliches Unterrichten schwierig (geworden) ist (Baumert et al. 2003; Solga/Wagner 2001). Inwieweit hier die Schulreformen der Bundesländer Abhilfe schaffen werden, bleibt abzuwarten, da sich die bisherige institutionelle Trennung (oder Segregation nach Schultypen) in eine schulinterne Segregation (nach Zweigen oder sogenannten "tracks“) oder in eine geografische Segregation (d.h. in sozial segregierte Nachbarschaften und damit segregierte Schulen wie in den USA) „übersetzen“könnte. 
In der bereits zitierten Studie ULME I (vgl. Lehmann et al. 2005) zeigt sich hinsichtlich der Kompetenzen zweierlei: In der Tat erreichten Jugendliche, die lediglich den Zugang zu einem teilqualifizierenden Berufsfachschulangebot gefunden hatten, durchschnittlich deutlich geringere Testwerte in Lesen, Mathematik und Englisch als die Jugendlichen in voll qualifizierenden Ausbildungsgängen (Lehmann et al. 2005, S. 59). Allerdings ist die Gruppe der Jugendlichen in den Berufsfachschulprogrammen nicht homogen. Sie zeichnet sich durch eine hohe Streuung (oder Varianz) in den vorhandenen Kompetenzen aus. Zudem existieren Überlappungen mit den Kompetenzbereichen der Jugendlichen in voll qualifizierenden Ausbildungsgängen (ebd., Abbildung 3.25).

Somit sind mehr Jugendliche „ausbildungsreif“ als angenommen, ihre vorhandenen Kompetenzen können jedoch mit den gegenwärtigen Rekrutierungspraxen und -kriterien nicht erkannt werden. Solche Praxen der Betriebe werden mit der sinkenden Zahl an Ausbildungssuchenden wohl zunehmend unter Druck geraten. Gleichwohl gibt es auch Jugendliche, die mehr Lernzeit benötigen und daher mit einer standardisierten Dauer von Ausbildungen und ohne zusätzliche Lernbegleitung Schwierigkeiten haben werden.

\section{Fazit: Eine Ausbildung für alle - aber wie?}

Wirtschaftlich, sozial und individuell kommt dem Erwerb eines beruflichen Ausbildungsabschlusses auf dem deutschen Arbeitsmarkt ein hoher Stellenwert zu. Junge Erwachsene ohne abgeschlossene Berufsausbildung (oder Studium) treten mit besonders schlechten Voraussetzungen in das Erwerbsleben ein - eine „(Langzeit-)Arbeitslosigkeitskarriere" ist für sie bereits früh im Lebensverlauf vorgezeichnet. Das deutsche Berufsbildungssystem ist dabei ein Ort sowohl für den Abbau als auch für die (Re-)Produktion von Ungleichheit. So werden an der Schwelle von der Schule in die Berufsausbildung soziale Ungleichheiten aus dem allgemeinbildenden Schulsystem fortgeschrieben und teilweise sogar noch verstärkt. Jugendliche mit maximal Hauptschulabschluss, oftmals aus sozial schwachen Familien, männlich und/oder mit Migrationshintergrund finden auch heute häufig nur Zugang zum sogenannten Übergangssystem.

Wie kann dies geändert werden? Zunächst sind ihre Lernbedingungen bereits im allgemeinbildenden Schulsystem zu verbessern, um die Schülerinnen und Schüler unter anderem besser für die steigenden Ansprüche der Ausbildungs- und Arbeitswelt vorzubereiten. Ziel der gegenwärtig stattfindenden Reformen im allgemeinbildenden Schulsystem sollte es daher sein, möglichst vielen Kindern und Jugendlichen den Erwerb eines mittleren Schulabschlusses zu ermöglichen (vgl. Expertenrat „Herkunft und Bildungserfolg“ 2011, S. 138; Solga 2012).
Allerdings wird es auch weiterhin Jugendliche geben, die dieses Ziel nicht erreichen. Damit es zukünftig besser gelingt, auch sie in voll qualifizierende Ausbildungsgänge zu integrieren, ist nach strukturellen Lösungen für das InsiderOutsider-Problem des deutschen Berufsbildungssystems zu suchen (siehe Abschnitt 1). Dem dualen System als dominanten Sektor des Berufsbildungssystems kommt hierbei eine zentrale Rolle zu. Hier entscheiden die Betriebe über die Besetzung der betrieblichen Ausbildungsplätze und somit über die weiteren Chancen der Jugendlichen. Von daher sind die Betriebe gefragt, ihre Rekrutierungspraxen zu überdenken und ggf. umzudenken. Dass auch andere Wege möglich sind, zeigen zahlreiche Modellprojekte, in denen über längerfristige Praktika und regelmäßige Praxistage eine Betriebsnähe für diese Jugendlichen hergestellt wurde, wodurch Betriebe die individuellen Potenziale von Jugendlichen mit einem Hauptschulabschluss (wieder) kennenlernen konnten (siehe Solga et al. 2012). Die (zumeist betrieblichen) Ausbildungschancen dieser Jugendlichen selbst wenn sie nach der 9. Klasse die Schule verlassen - sind dann erstaunlich hoch.

Zudem sind Veränderungen im Schulberufssystem notwendig, um die Integrationskraft des deutschen Ausbildungssystems zu stärken und den reinen Marktmechanismus abzuschwächen. Fehlende Ausbildungsplätze wurden bisher vor allem durch den Ausbau des Übergangssystems „ausgeglichen“, das jedoch nur wenig dazu beitragen kann, schlechte Ausgangsbedingungen beim Zugang zur voll qualifizierenden Ausbildung auszugleichen. Die Mittel, die für das Übergangssystem aufgebracht werden, könnten deutlich effizienter für die Schaffung zusätzlicher Ausbildungsplätze - insbesondere im Schulberufssystem - verwendet werden (siehe auch Beicht 2009, S. 15). Damit könnte einerseits der steigende Fachkräftebedarf in den Gesundheitsund Sozialberufen, für die im Schulberufssystem ausgebildet wird, besser gedeckt werden. Andererseits sollte auch - wie in Österreich (vgl. Ebner 2009) - stärker von der Möglichkeit Gebrauch gemacht werden, anerkannte Berufe des dualen Systems außerbetrieblich oder schulisch auszubilden (vgl. Lehmpfuhl/Müller-Tamke 2012; Solga 2009). Letzteres könnte einen wesentlichen Beitrag zur Verbesserung der Ausbildungschancen von Jugendlichen mit Migrationshintergrund leisten. Zudem wäre es sinnvoll, über eine Ausbildungsvergütung schulischer Ausbildungsangebote nachzudenken. Dies könnte zu einer größeren Geschlechtergleichheit der Ausbildungsbedingungen sowie zu einer abnehmenden Geschlechtersegregation in der Ausbildung beitragen, da so unter anderem die Attraktivität der zumeist schulischen (unvergüteten) Ausbildungsgänge in eher frauentypischen Berufen gesteigert werden kann.

Ferner sind Veränderungen in Ausbildungsablauf und -organisation erforderlich, damit Jugendlichen mit ungünstigen Lernausgangslagen nicht nur der Zugang zur Ausbildung gelingt, sondern auch, diese vollständig zu absolvieren. Dazu gehört eine Flexibilisierung von Ausbildungen, um 
Möglichkeiten zu schaffen, in einer verlängerten Ausbildungszeit Leistungsdefizite auszugleichen und den höheren Anforderungen von Berufen gerecht zu werden. Darüber hinaus könnten die Mittel, die derzeit für das Übergangssystem aufgewendet werden, für eine stärkere (personelle) Unterstützung von Betrieben bei der Ausbildung leistungsschwacher Jugendlicher effektiver eingesetzt werden.

Es gibt somit verschiedene Ansatzpunkte, die gemeinsam (jedoch nicht einzeln) zu einer Abmilderung der eingangs genannten Ausschlussmechanismen und damit zur Verbesserung der Integrationsfähigkeit des Berufsbildungssystems auch für gering vorgebildete Jugendliche sowie Jugendliche mit Migrationshintergrund beitragen können. Den Zugang zur Ausbildung allein dem „Markt“"zu überlassen, wäre angesichts des zentralen Stellenwerts beruflicher Ausbildungsabschlüsse für die Lebenschancen der jungen Erwachsenen sowie der wirtschaftlichen Bedarfe und Entwicklungen fatal.

\section{LITERATUR}

Autorengruppe Bildungsberichterstattung (2010): Bildung in Deutschland 2010, Bielefeld

Autorengruppe Bildungsberichterstattung (2012): Bildung in Deutschland 2012 Bielefeld

Baethge, M. (2008): Das berufliche Bildungswesen in Deutschland am Beginn des 21. Jahrhunderts, in: Cortina, K. S./Baumert, J./Leschinsky, A./Mayer, K. U./ Trammer, L. (Hrsg.): Das Bildungswesen in der Bundesrepublik Deutschland, Reinbek, S. 541-598

Baethge, M./Solga, H./Wieck, M. (2007): Bildung im Umbruch. Signale eines überfälligen Aufbruchs, Berlin, http://library.fes.de/pdf-files/stabsabteilung/04258/studie.pdf (letzter Zugriff: 6.8.2012)

Baumert, J./Trautwein, U./Artelt, C. (2003): Schulumwelten - institutionelle Bedingungen des Lehrens und Lernens, in: Artelt, C./Baumert, J./Klieme, E./ Neubrand, M./Prenzel, M./Schiefele, U./Schneider, W./Tillmann, K.-J./Weiß, M. (Hrsg.): PISA 2000 - Ein differenzierter Blick auf die Länder der Bundesrepublik Deutschland, Opladen, S. 261-333

Beicht, U. (2009): Verbesserung der Ausbildungschancen oder sinnlose Warteschleife?, Bundesinstitut für Berufsbildung, BIBB Report (11), Bonn

Beicht, U./Granato, M. (2010): Ausbildungsplatzsuche: Geringe Chancen für junge Frauen und Männer mit Migrationshintergrund, Bundesinstitut für Berufsbildung, BIBB Report (15), Bonn

Bertrand, M./Mullainathan, S. (2004): Are Emily and Greg More Employable than Lakisha and Jamal?, in: American Economic Review 94 (4), S. $991-1013$ Bundesinstitut für Berufsbildung (BIBB) (2011): Datenreport zum Berufsbildungsbericht 2011, Bonn

Bundesinstitut für Berufsbildung (BIBB) (2012): Datenreport zum Berufsbildungsbericht 2012, Bonn

Bundesministerium für Bildung und Forschung (BMBF) (2011): Berufsbildungsbericht 2011, Bonn

Dietz, M./Röttger, Ch./Szameitat, J. (2011): Betriebliche Personalsuche und Stellenbesetzungen: Neueinstellungen gelingen am besten über persönliche Kontakte, IAB-Kurzbericht (26), Nürnberg

Ebner, Ch. (2009): Neue Wege für die duale Berufsbildung - ein Blick auf Österreich, die Schweiz und Dänemark, WZBrief Arbeit (4), November, Berlin, http://www.wzb.eu/sites/default/files/publikationen/wzbrief/wzbriefarbeit042009_ebner.pdf (letzter Zugriff: 7.8.2012)

Expertenkommission Forschung und Innovation (EFI) (2009): Gutachten 2009. Jahresgutachten zu Forschung, Innovation und technologischer Leistungsfähigkeit Deutschlands 2009, Berlin

Expertenrat „Herkunft und Bildungserfolg” (2011): Empfehlungen für Bildungspolitische Weichenstellungen in der Perspektive auf das Jahr 2020 (BW2020), Stuttgart

Georg, W./Sattel, U. (2006): Berufliche Bildung, Arbeitsmarkt und Beschäftigung, in: Arnold, R./Lipsmeier, A. (Hrsg.): Handbuch der Berufsbildung, Wies baden, S. $125-152$

Gericke, N./Krupp, T./Troltsch, K. (2009): Unbesetzte Ausbildungsplätze - warum Betriebe erfolglos bleiben, BIBB-Report (10), Bonn
Giesecke, J./Ebner, C./Oberschachtsiek, D. (2010): Bildungsarmut und Arbeitsmarktexklusion, in: Hurrelmann, K./Quenzel, G. (Hrsg.): Bildungsverlierer: Neue Ungleichheiten, Wiesbaden, S. 421-438

Goldberg, A./Mourinho, D./Kulke, U. (1995): Arbeitsmarkt-Diskriminierung gegenüber ausländischen Arbeitnehmern in Deutschland, International Migration Papers (7), Genf

Koch, A. (1993): Notwendige Zukunftsinvestition, in: W\&S, Zeitschrift für Wirtschaftliche und Soziale Bildung (6), S. 8-10

Kohlrausch, B./Solga, H. (2012): Übergänge in die Ausbildung: Welche Rolle spielt die "Ausbildungsreife"?, in: Zeitschrift für Erziehungswissenschaft (4) (im Erscheinen)

Krekel, E. M./Ulrich, J. G. (2009): Jugendliche ohne Berufsabschluss, Berlin, http://library.fes.de/pdf-files/stabsabteilung/06430.pdf (letzter Zugriff: 6.8.2012) Lehmann, R. H./Ivanov, S./Hunger, S./Gänsfuß, R. (2005): ULME I. Untersuchung der Leistungen, Motivationen und Einstellungen zu Beginn der beruflichen Ausbildung, Hamburg

Lehmann, R. H./Seeber, S./Hunger, S. (2006): ULME II. Untersuchung der Leis tungen, Motivationen und Einstellungen der Schülerinnen und Schüler in den Abschlussklassen der teilqualifizierenden Berufsfachschulen, Hamburg Lehmpfuhl, U./Müller-Tamke, W. (2012): Schulische Berufsausbildung mit Kammerabschluss gemäß § 43 Abs. 2 Berufsbildungsgesetzt (BBiG), in: Berufsbildung in Wissenschaft und Praxis (1), S. 43-46

Müller, W./Shavit, Y. (1998): Bildung und Beruf im institutionellen Kontext, in: Zeitschrift für Erziehungswissenschaften (1), S. 501-533

Nationaler Pakt für Ausbildung und Fachkräftenachwuchs in Deutschland (2010), Berlin, 19.6.2009, http://www.arbeitsagentur.de/zentraler-Content/A04Vermittlung/A041-Erschliessung/Publikation/pdf/Ausbildungsreife-Berufsorientierung.pdf (letzter Zugriff: 1.5.2012)

Nationaler Pakt für Ausbildung und Fachkräftenachwuchs in Deutschland (2012): Gemeinsame Pressemitteilung „Erstes Jahr des erneuerten Ausbildungspaktes: Bessere Chancen auf eine Berufsausbildung" vom 1.2., http:// www.bmwi.de/DE/Presse/pressemitteilungen, did=473970.html (letzter Zugriff: 7.8.2012)

Organisation for Economic Co-operation and Development (OECD) (2000): Literacy in the Information Age, Paris

Organisation for Economic Co-operation and Development (OECD) (2010):

Off to a Good Start? Jobs for Youth, Paris

Organisation for Economic Co-operation and Development (OECD) (2011):

Education at a Glance, OECD Indicators, Paris

Pötter, U./Prein, G./Steiner, Ch. (2012): Geförderte Chancen? Der Beitrag der Ausbildungsförderung für den Berufseinstieg im "Laboratorium Ostdeutschland“, in: Becker, R./Solga, H. (Hrsg.): Soziologische Bildungsforschung. Sonderheft der Kölner Zeitschrift für Soziologie und Sozialpsychologie (52), Wiesbaden, S. 234-255

Pressemitteilung zum Ausbildungspakt 2009, vom 13.10., Berlin, http://www. bmwi.de/BMWi/Navigation/Presse/pressemitteilungen,did=316786.html (letzter Zugriff: 1.5.2012)

Protsch, P. (2011): Zugang zu Ausbildung. Eine historisch vergleichende Perspektive auf den segmentierten Ausbildungsmarkt in (West-)Deutschland, WZB Discussion Paper SP I 2011 -502, Berlin, http://bibliothek.wz-berlin.de/pdf/2011/ i11-502.pdf (letzter Zugriff: 30.4.2012)

Protsch, P./Dieckhoff, M. (2011): What Matters in the Transition from School to VocationalTraining in Germany: Educational Credentials, Cognitive Abilities or Personality?, in: European Societies 13 (1), S. 69-91

Protsch, P./Dieckhoff, M. (2012): Noten, kognitive Fähigkeiten oder Persönlichkeit: Was bei der Suche nach einem Ausbildungsplatz zählt, WZBrief Bildung (16), Berlin, http://www.wzb.eu/sites/default/files/publikationen/wzbrief/ wzbriefbildung_162011_protsch_dieckhoff_0.pdf (Zugriff: 1.5.2012)

Reißig, B./Gaupp, N./Lex, T. (2008a): Vorwort, in: Dies. (Hrsg.): Hauptschüler auf dem Weg von der Schule in die Arbeitswelt, München, S. 7-19

Reißig, B./Gaupp, N./Lex, T. (2008b): Übergangswege von Hauptschulabsolventinnen und -absolventen aus der Schule in die Ausbildung, in: Dies. (Hrsg.): Hauptschüler auf dem Weg von der Schule in die Arbeitswelt, München, S. 58-81

Schnur, P./Zika, G. (2007): Arbeitskräftebedarf bis 2025, IAB-Kurzbericht (26), Nürnberg

Seyfried, B. (2006): Berufsausbildungsvorbereitung aus betrieblicher Sicht, Bonn

Solga, H. (2005): Ohne Abschluss in die Bildungsgesellschaft. Die Erwerbschancen gering qualifizierter Personen aus ökonomischer und soziologischer Perspektive, Opladen 
Solga, H. (2009): Der Blick nach vorn: Herausforderungen an das deutsche Ausbildungssystem, WZB Discussion Paper SP I 2009-507, Berlin, http:// bibliothek.wz-berlin.de/pdf/2009/i09-507.pdf (letzter Zugriff: 30.4.2012

Solga, H. (2011): Bildungsarmut und Ausbildungslosigkeit in der Bildungsund Wissensgesellschaft, in: Becker, R. (Hrsg.): Lehrbuch der Bildungssoziologie, Wiesbaden, S. $411-448$

Solga, H. (2012): Bildung und materielle Ungleichheiten - Der investive Sozialstaat auf dem Prüfstand, in: Becker, R./Solga, H. (Hrsg.): Soziologische Bildungsforschung. Sonderheft der Kölner Zeitschrift für Soziologie und Sozialpsychologie (52), Wiesbaden, S. 459-487

Solga, H./Baas, M./Kohlrausch, B. (2012): Mangelnde Ausbildungsreife Hemmnis bei der Lehrstellensuche von Jugendlichen mit Hauptschulabschluss? WZBrief Bildung 19/2012, Berlin, http://bibliothek.wzb.eu/wzbriefbildung/WZBriefBildung192011_solga_baas_kohlrausch.pdf, (letzter Zugriff: 30.4.2012)

Solga, H./Wagner, S. (2001): Paradoxie der Bildungsexpansion: Die doppelte Benachteiligung von Hauptschülern, in: Zeitschrift für Erziehungswissenschaft (4), S. 107-127

Statistisches Bundesamt (2011a): Fachserie 1, Reihe 2.2. Bevölkerung mit Migrationshintergrund, Wiesbaden.

Statistisches Bundesamt (2011b): Fachserie 11, Reihe 2. Berufliche Schulen, Schuljahr 2010/2011, Wiesbaden

Uhlig, J./Solga, H./Schupp, J. (2009): Bildungsungleichheiten und blockierte Lernpotenziale, in: Zeitschrift für Soziologie 38 (5), S. 418-441
Uhlig, J. (2010): Brachliegende Potenziale durch Underachievement, WZBrief Bildung 12/2010, Berlin, http://www.wzb.eu/sites/default/files/publikationen/ wzbrief/wzbriefbildung_122010_uhlig_0.pdf (letzter Zugriff: 1.5.2012).

\section{AUTORINNEN}

HEIKE SOLGA , Direktorin der Abteilung „Ausbildung und Arbeitsmarkt" am Wissenschaftszentrum Berlin für Sozialforschung (WZB) und Professorin für Soziologie an der Freien Universität Berlin. Arbeitsschwerpunkte: Arbeitsmarkt, Bildung und Ausbildung.

@ heike.solga@wzb.eu

LAURA MENZE, Wissenschaftliche Mitarbeiterin in der Projektgruppe "Nationales Bildungspanel: Berufsbildung und lebenslanges Lernen“ am WZB. Arbeitsschwerpunkte: Arbeit und Arbeitsmarkt, Bildung und Ausbildung, Sozialstaat und soziale Ungleichheit.

@ laura.menze@wzb.eu 